\title{
On the Calculation of 2D Green's Functions Via Sommerfeld Integrals and the Spatial Singularity Method
}

\author{
Said Mikki ${ }^{1}$ \\ ${ }^{1}$ Department of ECECS, University of New Haven, CT, USA
}

\begin{abstract}
We provide a low-level review of the computation of Sommerfeld integration theory using the singularity expansion method (SEM) to analytically estimate the short-wavelength components of the 2dimensional Green's function. The SEM is employed to replace the infinite tail of the spectral integral by a closed-form evaluation. The various steps in the SEM substitution and the calculations are elaborately presented and discussed with emphasis on giving the missing details often not included in the published literature.
\end{abstract}

\section{Introduction}

Sommerfeld integrals arise in the problem of calculating Green's functions of electromagnetic sources in media containing open boundaries. For a source above stratified media, the spectral-domain Green's functions can be obtained in closed form. However, in order to obtain the corresponding spatial-domain Green's functions it is required to calculate the inverse Fourier transform. This process results in what is known as Sommerfeld integrals. The calculation of these integrals is very difficult due to the oscillatory nature of the integrand and the existence of branch points and surface pole (SWP) singularities. Discrete complex image theory (DCIT) or the spatial singularity expansion method (SEM) is a fast and efficient method that can be used to treat such problems [1-6]. Applications include computing the electromagnetic fields in cylindrical nanostructures $[7,8]$, RF antenna system analysis and design [9], and near-field nano-optics [10].

\section{Formulation of the Problem}

In what follows, we assume familiarity with the concept of Green's function at the level of [11]. Consider the following spatial domain 2D Green's functions calculated using the inverse Hankel transform

$$
G(\rho)=\frac{1}{2} \int_{-\infty}^{\infty} d k_{\rho} k_{\rho} J_{0}\left(k_{\rho} \rho\right) \tilde{G}\left(k_{\rho}\right)
$$

where $\tilde{G}\left(k_{\rho}\right)$ is the spectral-domain function. For review of the bessel functions $J_{0}(x)$ and the derivation of (1), see [11]. If the function $\tilde{G}\left(k_{\rho}\right)$ is even then we can write the above integral as

$$
G(\rho)=\int_{0}^{\infty} d k_{\rho} k_{\rho} J_{0}\left(k_{\rho} \rho\right) \tilde{G}\left(k_{\rho}\right)
$$

The propagation constant $k_{z}$ is given by

$$
k_{z}=\sqrt{k_{i}-k_{\rho}}
$$

where $k_{i}$ is the wavenumber in the $i$ th medium. We now present the computation of the spectral integral above in the following hierarchical method. 
II.a 1-Level Approach: The one-level approach consists of the following contour

$$
C_{k_{z}}: k_{z}=k_{i}\left[-j t+\left(1-\frac{t}{T_{0}}\right)\right] \quad 0 \leq t \leq T_{0}
$$

Fig. 1 shows how this contour is mapped from the complex $k_{z}$ plane to the complex $k_{\rho}$. Here, we have

$$
k_{\rho_{\max }}=k_{i} \sqrt{1+T_{0}^{2}}
$$

Thus, to insure that the deformed path $C_{\rho}$ will include all the branch points, the surface wave poles (SWP), and the asymptotic behavior of the spectral domain GF when $k_{\rho} \rightarrow \infty$, it is important to choose $k_{\rho_{\max }}$ large enough.

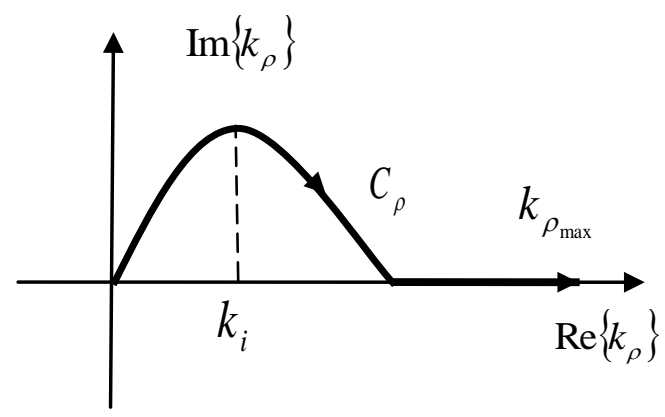

(a)

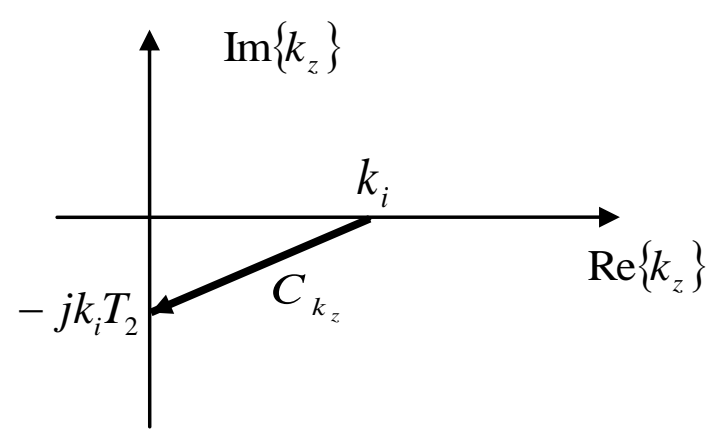

(b)

Fig. 1 1-level integration path in a) the $k_{\rho}$ plane b) $k_{z}$ plane

We will now approximate $j k_{z} \tilde{G}_{C_{\rho}}\left(k_{\rho}\right)$ using the singularity expansion method (SEM), powered by Prony's Method (PM) or the Generalized Pencil of Function (GPOF) Method

$$
j k_{z} \tilde{G}_{C_{\rho}}\left(k_{\rho}\right) \approx \sum_{n=1}^{N} b_{n} e^{\beta_{n} t}
$$

The reason of including the multiplicative factor $j k_{z}$ in equation (6) will be apparent when we use Sommerfeld identity later. Alternatively, the equation (6) can be re-written in the following form 


$$
\tilde{G}_{C_{\rho}}\left(k_{\rho}\right)=\sum_{n=1}^{N} b_{n} \frac{e^{\beta_{n} t}}{j k_{z}}=\sum_{n=1}^{N} a_{n} \frac{e^{-\alpha_{n} k_{z}}}{j k_{z}}
$$

where it is easy to show that

$$
\alpha_{n}=\frac{\beta_{n} T_{0}}{k_{i}\left(1+j T_{0}\right)} \quad a_{n}=b_{n} e^{k_{i} \alpha_{n}}
$$

Substituting (7) into (2) we get

$$
\begin{aligned}
G(\rho) & =\int_{0}^{\infty} d k_{\rho} k_{\rho} J_{0}\left(k_{\rho} \rho\right) \sum_{n=1}^{N} a_{n} \frac{e^{-\alpha_{n} k_{z}}}{j k_{z}} \\
& =\sum_{n=1}^{N} a_{n} \int_{0}^{\infty} d k_{\rho} k_{\rho} J_{0}\left(k_{\rho} \rho\right) \frac{e^{-\alpha_{n} k_{z}}}{j k_{z}}
\end{aligned}
$$

The following form of Sommerfeld identity will now be used

$$
\frac{e^{-j k r}}{r}=\int_{0}^{\infty} d k_{\rho} k_{\rho} J_{0}\left(k_{\rho} \rho\right) \frac{e^{-j k_{z}|z|}}{j k_{z}}
$$

Equation (9) become then

$$
G(\rho)=\sum_{n=1}^{N} a_{n} \frac{e^{-j k r_{n}}}{r_{n}}
$$

where we have

$$
r_{n}=\sqrt{\rho^{2}-\alpha_{n}^{2}}
$$

which is the distance - generally complex - of the $n$th image.

II.b 2-Level Approach: The two-level DCIT approach consists of sampling over the following two parameterized integration paths

$$
\begin{array}{ll}
C_{1}: k_{z}=-j k_{i}\left[T_{2}+t_{1}\right] & 0 \leq t_{1} \leq T_{1} \\
C_{2}: k_{z}=k_{i}\left[-j t+\left(1-\frac{t_{2}}{T_{2}}\right)\right] & 0 \leq t_{2} \leq T_{2}
\end{array}
$$

The corresponding path in the $k_{\rho}$ complex plane is shown in Fig. 1. It is clear that a linear variation in the $k_{z}$ plane shown in Fig. 1(a) is translated in the $k_{\rho}$ plane to the deformed path shown in Fig. 1(b). Notice that $k_{\rho_{\max }}=k_{i} \sqrt{1+T_{2}^{2}}$. 


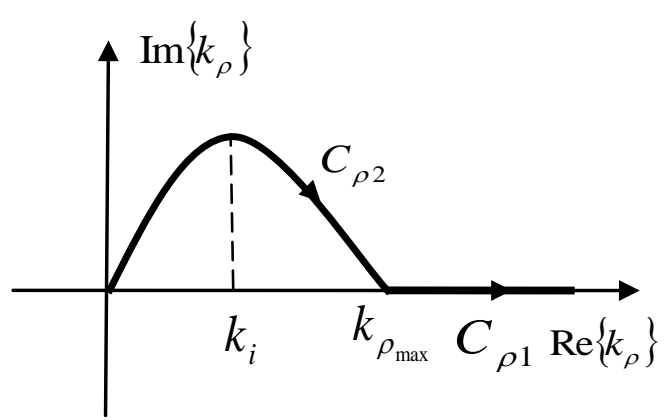

(a)

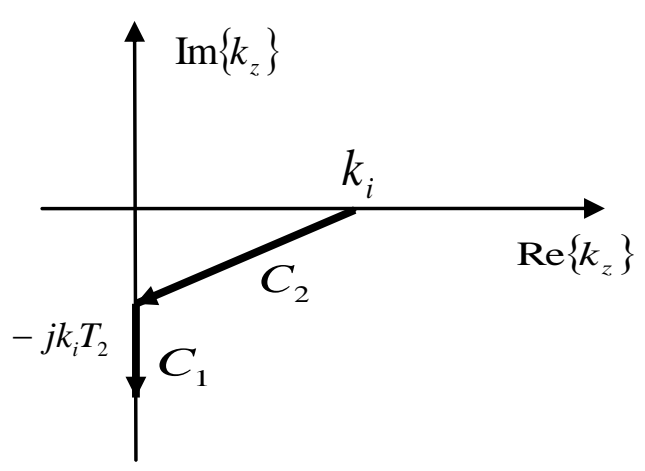

(b)

Fig. 2 2-level Integration path in a) the $k_{\rho}$ plane b) $k_{z}$ plane

The Prony's method (PM), or the Generalized Pencil of Function (GPOF) method, can be utilized to expand an arbitrary function in terms of sum of complex exponentials. We utilize now this method in order to calculate the Sommerfeld integral of equation (2).

First, approximate the "tail" region of the spectral domain function, i.e. the region over the contour $C_{\rho 1}$, in the following way

$$
j k_{z} \tilde{G}_{C_{\rho 1}}\left(k_{\rho}\right) \approx \sum_{n=1}^{N_{1}} b_{1 n} e^{\beta_{1 n} t_{1}}
$$

Next, putting the above in a form suitable for the future use of Sommerfeld identity, we write

$$
\tilde{G}_{C_{\rho 1}}\left(k_{\rho}\right)=\sum_{n=1}^{N_{1}} b_{1 n} \frac{e^{\beta_{1 n} t_{1}}}{j k_{z}}=\sum_{n=1}^{N_{1}} a_{1 n} \frac{e^{-\alpha_{1 n} k_{z}}}{j k_{z}}
$$

where it easy to find that

$$
\alpha_{1 n}=\frac{\beta_{1 n}}{j k_{i}} \quad, a_{1 n}=b_{1 n} e^{-j k_{i} \alpha_{1 n} T_{2}}
$$

Next, the function approximated in (15) is subtracted from the original spectral domain function $\tilde{G}\left(k_{\rho}\right)$. The result will be non-zero only on the contour $C_{\rho 2}$. The total integration can be then written as 


$$
\tilde{G}\left(k_{\rho}\right)=\int_{C_{\rho 2}} d k_{\rho} k_{\rho} J_{0}\left(k_{\rho} \rho\right)\left[\tilde{G}\left(k_{\rho}\right)-\tilde{G}_{C_{\rho 1}}\left(k_{\rho}\right)\right]+\int_{C_{\rho 1}+C_{\rho 2}} d k_{\rho} k_{\rho} J_{0}\left(k_{\rho} \rho\right) \tilde{G}_{C_{\rho 1}}\left(k_{\rho}\right)
$$

Now, the spectral domain function in the first integral is approximated using PM or GPOF over the path $C_{\rho 2}$ as follow

$$
j k_{z}\left[\tilde{G}\left(k_{\rho}\right)-\tilde{G}_{C_{\rho 1}}\left(k_{\rho}\right)\right] \approx \sum_{n=1}^{N_{2}} b_{2 n} e^{\beta_{2 n} t_{2}}
$$

where this yield

$$
\tilde{G}\left(k_{\rho}\right)-\tilde{G}_{C_{\rho 1}}\left(k_{\rho}\right)=\sum_{n=1}^{N_{2}} b_{2 n} \frac{e^{\beta_{2 n} t_{2}}}{j k_{z}}=\sum_{n=1}^{N_{2}} a_{2 n} \frac{e^{-\alpha_{2 n} k_{z}}}{j k_{z}}
$$

and again it is easy to deduce that

$$
\alpha_{2 n}=\frac{\beta_{2 n} T_{2}}{k_{i}\left(1+j T_{2}\right)} \quad, a_{2 n}=b_{2 n} e^{k_{i} \alpha_{2 n}}
$$

By substituting into equation (17) the approximations (15) and (19) and utilizing Sommerfeld identity in (10) we get

$$
\begin{gathered}
G(\rho)=\sum_{n=1}^{N_{1}} a_{1 n} \frac{e^{\alpha_{1 n} r_{1 n}}}{r_{1 n}}+\sum_{n=1}^{N_{2}} a_{2 n} \frac{e^{\alpha_{2 n} r_{2 n}}}{r_{2 n}} \\
r_{(1,2) n}=\sqrt{\rho^{2}-\alpha_{(1,2) n}^{2}}
\end{gathered}
$$

\section{III.c 3-Level Formulation}

The 3-level formulation follows the basic idea developed in the previous section. Fig. 3 illustrates the three paths

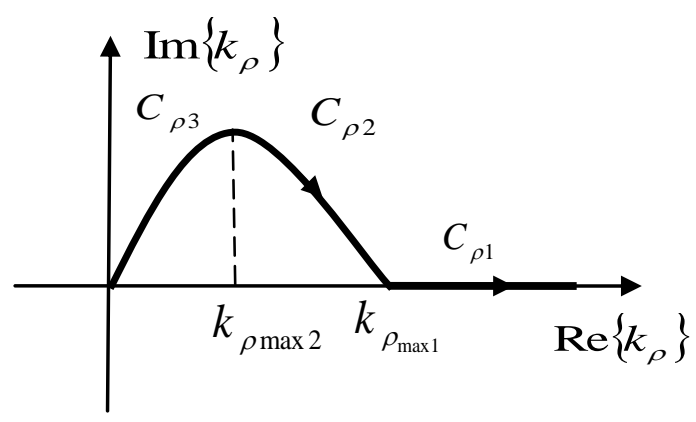

(a) 


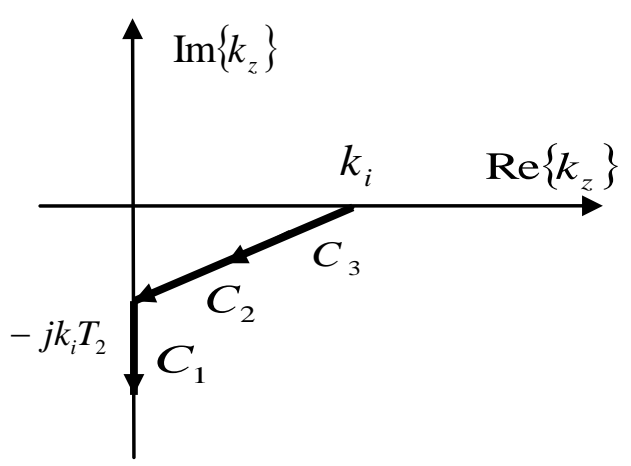

(b)

Fig. 3 3-level Integration path in a) the $k_{\rho}$ plane b) $k_{z}$ plane

The integration path in the region $\left[0, k_{\rho \max 1}\right]$ is divided into two parts, $\left[0, k_{\rho \max 2}\right]$ and $\left[k_{\rho \max 2}, k_{\rho \max 1}\right]$. The reason of doing this is the fact that for some problems the spectral domain Green's functions may vary considerably in one region while being smooth in other regions. Since the PM or the GPOM work only for uniform sampling, they can not be utilized directly in sampling the function unless a huge number of points is used (may be thousands). To avoid this, we divide the main region $\left[0, k_{\rho \max 1}\right]$ into two sub-regions where higher number of samples will be used in each region. Because the sub-regions are smaller than the original interval, the "higher" number of samples here is still less what would be required if the entire region is sampled once using uniform sampling. The suggested contour path will be given in the following way

$$
\begin{array}{ll}
C_{1}: k_{z}=-j k_{i}\left[T_{2}+t_{1}\right] & , 0 \leq t_{1} \leq T_{1} \\
C_{2}: k_{z}=k_{i}\left[-j\left(t_{2}\right)+\left(1-\frac{t_{2}}{T_{2}}\right)\right] & , T_{3} \leq t_{2} \leq T_{2} \\
C_{3}: k_{z}=k_{i}\left[-j t_{3}+\left(1-\frac{t_{3}}{T_{2}}\right)\right] & , 0 \leq t_{3} \leq T_{3}
\end{array}
$$

We first approximate the spectral domain function in the first region as follow

$$
f_{1}\left(k_{\rho}\right)=\tilde{G}_{C_{\rho 1}}\left(k_{\rho} \rho\right)=\sum_{n=1}^{N_{1}} b_{1 n} \frac{e^{\beta_{1 n} t_{1}}}{j k_{z}}=\sum_{n=1}^{N_{1}} a_{1 n} \frac{e^{-\alpha_{1 n} k_{z}}}{j k_{z}} \quad, k_{\rho} \in\left[0, k_{\rho \max 2}\right]
$$

where $\alpha_{1 n}$ and $a_{1 n}$ are given by equations (16).

Next, we approximate the following function in the interval $\left[k_{\rho \max 2}, k_{\rho \max 1}\right]$ as follow

$$
f_{2}\left(k_{\rho}\right)=\tilde{G}_{C_{\rho 2}}\left(k_{\rho}\right)=\sum_{n=1}^{N_{2}} b_{2 n} \frac{e^{\beta_{2 n} t_{2}}}{j k_{z}}=\sum_{n=1}^{N_{2}} a_{2 n} \frac{e^{-\alpha_{2 n} k_{z}}}{j k_{z}} \quad, k_{\rho} \in\left[k_{\rho \max 2}, k_{\rho \max 1}\right]
$$

where $\alpha_{2 n}$ and $a_{2 n}$ are given by (20). 
We now approximate the following function over the interval $\left[k_{\rho \max 2}, \infty\right]$

$$
f_{3}\left(k_{\rho}\right)=\tilde{G}\left(k_{\rho}\right)-f_{1}\left(k_{\rho}\right)-f_{2}\left(k_{\rho}\right)=\sum_{n=1}^{N_{3}} b_{3 n} \frac{e^{\beta_{3 n} t_{2}}}{j k_{z}}=\sum_{n=1}^{N_{3}} a_{3 n} \frac{e^{-\alpha_{3 n} k_{z}}}{j k_{z}} \quad, k_{\rho} \in\left[0, k_{\rho \max 2}\right]
$$

where $\alpha_{2 n}$ and $a_{2 n}$ are also given by (20).

The inverse Hankel transformation can be written then as

$$
\tilde{G}\left(k_{\rho}\right)=\int_{C_{\infty}} d k_{\rho} k_{\rho} J_{0}\left(k_{\rho} \rho\right)\left[\tilde{G}\left(k_{\rho}\right)-f_{2}\left(k_{\rho}\right)-f_{1}\left(k_{\rho}\right)\right]+\int_{C_{\infty}} d k_{\rho} k_{\rho} J_{0}\left(k_{\rho} \rho\right)\left[f_{1}\left(k_{\rho}\right)+f_{2}\left(k_{\rho}\right)\right]
$$

Now, since

$$
\tilde{G}\left(k_{\rho}\right)-f_{1}\left(k_{\rho}\right)-f_{2}\left(k_{\rho}\right)=0 \quad, k_{\rho} \in\left[0, k_{\rho \max 2}\right]
$$

we can write (27) as

$$
G(\rho)=\int_{C_{3}} d k_{\rho} k_{\rho} J_{0}\left(k_{\rho} \rho\right)\left[\tilde{G}\left(k_{\rho}\right)-f_{2}\left(k_{\rho}\right)-f_{1}\left(k_{\rho}\right)\right]+\int_{C_{\infty}} d k_{\rho} k_{\rho} J_{0}\left(k_{\rho} \rho\right)\left[f_{1}\left(k_{\rho}\right)+f_{2}\left(k_{\rho}\right)\right]
$$

which is the Green's function in the spatial domain expressed in terms of mixed spectral and closed-form analytical expression.

\section{Conclusion}

We provided a pedagogically illuminating reexamination of how to compute Sommerfeld integrals using the singularity expansion method. The derivation was given with high degree of details to explain how each integration interval was handled in the spectral domain. The expression (28) is much faster to evaluate than a fully-fledged numerical calculation over the entire spectral domain. The reduction of cost clearly comes from the fact that the shortwavelength components have been approximated analytically using the deeper SEM poles and residues information buried in the high spatial frequency band. Physically, it appears that the SEM data (poles and residues) represent special spectral points of major singularities that capture the overall (global) behavior of the Green's functions [12].

\section{References}

[1] D. C. Fang, J. J. Yang, and G. Y. Delisle, "Discrete image theory for horizontal electric dipoles in a multilayered medium”, Proc. Inst. Elect. Eng. Pt. H, vol. 135, pp. 297-303, oct. 1988.

[2] Y. L. Chow, J. J. Yang, D. G. Fang, and G. E. Howard, “A closed-form spatial Green's function for the thick microstrip substrate," IEEE Trans. Microw. Theory Tech., vol. 39, no. 3, pp. 558-592, Mar. 1991.

[3] M. I. Aksun and R. Mittra, "Derivation of closed-form Green's functions for a general microstrip geometry,” IEEE Trans. Microw. Theory Tech., vol. MTT-40, no. 11, pp. 2055-2062, Nov. 1992.

[4] M. I. Aksun, "A robust approach for the derivation of closed-form Green's functions," IEEE Trans. Microw. Theory Tech., vol. 44, no. 5, pp. 654-658, May. 1996. 
[5] Noyan Kmayman and M. I. Aksun, "Efficient use of closed-form Green's Functions for the analysis of planar geometries with vertical connections", IEEE Trans. Microw. Theory Tech., vol. MTT-45, no. 5, pp. 593-603, May 1997.

[6] M. I. Aksun and Gulbin Dural, "Clarification of issues on the closed-form Green's functions in stratified media,” IEEE Trans. Antennas Propag., vol. 53, no. 11, pp. 3644-3653, November. 2005.

[7] S. M. Mikki and A. A. Kishk, "Theory of optical scattering by carbon nanotubes," Microwave and Optical Technology Letters 49 (10), 2360-2364, 2007.

[8] S. M. Mikki and A. A. Kishk, "Electromagnetic Scattering by Multi-Wall Carbon Nanotubes," Progress In Electromagnetics Research B, Vol. 17, 49-67, 2009.

[9] C. Balansi, Antenna Theory: Analysis and Design, Wiley, 2016.

[10] L. Novonty and B. Hecht, Principles of Nano-Optics, Cambridge University Press, 2012.

[11] C. Balanis, Advanced Engineering Electromagnetics, Wiley, 1989.

[12] S. Mikki, A. M. Alzahed and Y. M. M. Antar, "The Spatial Singularity Expansion Method for Electromagnetics," in IEEE Access, vol. 7, pp. 124576-124595, 2019. 